\title{
Neutralizing antibodies to interferon beta: Assessment of their clinical and radiographic impact: An evidence report
}

\section{Report of the Therapeutics and Technology Assessment Subcommittee of the American Academy of Neurology}

\author{
D.S. Goodin, MD; E.M. Frohman, MD, FAAN; B. Hurwitz, MD; P.W. O'Connor, MD; \\ J.J. Oger, MD, FRCPC, FAAN; A.T. Reder, MD; and J.C. Stevens, MD, FAAN
}

\begin{abstract}
The clinical and radiologic impact of developing neutralizing antibodies (NAbs) to interferon beta (IFN $\beta$ ) while on this therapy for multiple sclerosis (MS) is assessed. On the basis of Class II and III evidence, it is concluded that treatment of patients with MS with IFN $\beta$ (Avonex, Betaseron, or Rebif) is associated with the production of NAbs (Level A). NAbs in the serum are probably associated with a reduction in the radiographic and clinical effectiveness of IFN $\beta$ treatment (Level B). In addition, the rate of NAb production is probably less with IFN $\beta-1$ a treatment than with IFN $\beta-1 b$ treatment, although the magnitude and persistence of this difference is difficult to determine (Level B). Finally, it is probable that there is a difference in seroprevalence due to variability in the dose of IFN $\beta$ injected or in the frequency or route of its administration (Level B). Regardless of the explanation, it seems clear that IFN $\beta$-1a (as it is currently formulated for IM injection) is less immunogenic than the current IFN $\beta$ preparations (either IFN $\beta-1 \mathrm{a}$ or IFN $\beta$-1b) given multiple times per week subcutaneously (Level A). However, because NAbs disappear in some patients even with continued IFN $\beta$ treatment (especially in patients with low titers), the persistence of this difference is difficult to determine (Level B). Although the finding of sustained high-titer NAbs (>100 to $200 \mathrm{NU} / \mathrm{mL}$ ) is associated with a reduction in the therapeutic effects of IFN $\beta$ on radiographic and clinical measures of MS disease activity, there is insufficient information on the utilization of NAb testing to provide specific recommendations regarding when to test, which test to use, how many tests are necessary, or which cutoff titer to apply (Level U).
\end{abstract}

NEUROLOGY 2007;68:977-984

The development of neutralizing antibodies (NAbs) to proteins administered therapeutically is often associated with a reduction in the biologic actions that these proteins exert. It is therefore surprising that

Additional material related to this article can be found on the Neurology Web site. Go to www.neurology.org and scroll down the Table of Contents for the March 27 issue to find the title link for this article. the clinical and radiographic impact of NAbs to interferon beta (IFN $\beta$ ) in the treatment of multiple sclerosis (MS) is controversial. This assessment evaluates the clinical and radiographic impact of NAbs in this setting and considers some of the difficulties in this research area that may explain the ongoing controversy. In this regard, it is useful for readers to appreciate the complexity of this particular biologic system..$^{1-9}$ Thus, a brief overview of IFN biology is provided in the supplementary material to this as-

From the University of California, San Francisco (D.S.G.); University of Texas Southwestern (E.M.F.), Dallas; Duke University Medical College (B.H.), Durham, NC; St. Michaels Hospital (P.W.O.), Toronto, Ontario, Canada; University of BC (J.J.O.), Vancouver, British Columbia, Canada; The University of Chicago (A.T.R.), Oak Park, IL; and Lutheran Medical Office (J.C.S.), Fort Wayne, IN.

Disclosure: The authors report no conflicts of interest.

Received August 10, 2006. Accepted in final form December 7, 2006.

Approved by the Therapeutics and Technology Subcommittee on July 28, 2006; by the Practice Committee on November 11, 2006; and by the AAN Board of Directors on January 4, 2007.

Address correspondence and reprint requests to the American Academy of Neurology, 1080 Montreal Avenue, St. Paul, MN 55116; e-mail: guidelines@aan.com 
Table 1 Effect of NAbs on clinical and MRI outcomes in MS therapeutic trials

\begin{tabular}{|c|c|c|c|c|c|c|c|c|}
\hline Study & $\mathrm{n}$ & Year & $\begin{array}{l}\text { Clinical } \\
\text { activity }\end{array}$ & $\begin{array}{l}\text { Clinical } \\
\text { severity }\end{array}$ & $\begin{array}{c}\text { MRI } \\
\text { activity }\end{array}$ & $\begin{array}{c}\text { MRI } \\
\text { severity }\end{array}$ & Drug & Class \\
\hline IFN $\beta-1 b$ MS Study Group (low dose) ${ }^{14-16,32 *}$ & 55 & 1996 & $-(\mathrm{NS})$ & & $-(\mathrm{NS})$ & & IFN $\beta-1 b$ & II \\
\hline IFN $\beta-1 b$ MS Study Group (high dose) $)^{14-16,32 *}$ & 53 & 1996 & $+\dagger$ & $-(\mathrm{NS})$ & $+(\mathrm{NS})$ & $+(\mathrm{NS})$ & IFN $\beta-1 b$ & II \\
\hline $\operatorname{MSCRG}^{17,18,28}$ & 23 & 1998 & $-(\mathrm{NS})$ & $+(\mathrm{NS})$ & $+(\mathrm{NS})$ & & IFN $\beta-1 \mathrm{a}$ & II \\
\hline PRISMS (low dose) $)^{19,21,22,35 *}$ & 55 & 2001 & $+(\mathrm{NS})$ & $+\dagger^{\mathrm{a}}$ & $+\S$ & $+\S$ & IFN $\beta-1 \mathrm{a}$ & II \\
\hline PRISMS (high dose) $)^{19,21,22,35 *}$ & 35 & 2001 & $+\ddagger$ & & $+\S$ & $+\S$ & IFN $\beta-1 \mathrm{a}$ & II \\
\hline SPECTRIMS (low dose) ${ }^{23,} 24 *$ & 43 & 2001 & $-(\mathrm{NS})$ & $-(\mathrm{NS})$ & & & IFN $\beta-1 \mathrm{a}$ & II \\
\hline SPECTRIMS (high dose) $)^{23,24 *}$ & 30 & 2001 & $+(\mathrm{NS})$ & $-(\mathrm{NS})$ & & & IFN $\beta-1 \mathrm{a}$ & II \\
\hline INCOMIN $^{25,39}$ & 28 & 2002 & $-(\mathrm{NS})$ & & $+(\mathrm{NS})$ & & IFN $\beta-1 \mathrm{a} / 1 \mathrm{~b}$ & II \\
\hline EVIDENCE $^{26,27}$ & 91 & 2002 & $+(\mathrm{NS})$ & & & & IFN $\beta-1 a$ & II \\
\hline European SPMS IFN $\beta-1 b^{20,} 29 *$ & 95 & 2003 & $+\ddagger$ & & & & IFN $\beta-1 b$ & II \\
\hline Sorensen et al. ${ }^{30 *}$ & 169 & 2003 & $+\ddagger$ & $+(\mathrm{NS})$ & & & IFN $\beta-1 a / 1 b$ & III \\
\hline Frank et al. ${ }^{31 *}$ & 11 & 2004 & & & & & IFN $\beta-1 b$ & II \\
\hline North American SPMS IFN $\beta-1 b$ Study Group ${ }^{33 *}$ & 174 & 2004 & $+\dagger$ & & $?(\mathrm{NS})$ & $?(\mathrm{NS})$ & IFN $\beta-1 b$ & II \\
\hline Malucchi et al. ${ }^{40}$ & 13 & 2004 & $+\dagger^{\mathrm{b}}$ & & & & IFN $\beta-1 \mathrm{a} / 1 \mathrm{~b}$ & III \\
\hline Perini et al. $^{41}$ & 22 & 2004 & $+(\mathrm{NS})^{\mathrm{b}}$ & $?(\mathrm{NS})^{\mathrm{b}}$ & & & IFN $\beta-1 a / 1 b$ & III \\
\hline Eur. Dose Comparison Study (low dose) ${ }^{37,38 *}$ & 7 & 2005 & $+\dagger 1$ & $(\mathrm{NS})$ & $+(\mathrm{NS})$ & & IFN $\beta-1 \mathrm{a}$ & II \\
\hline Eur. Dose Comparison Study (high dose) ${ }^{37,38 *}$ & 19 & 2005 & & & & & IFN $\beta-1 \mathrm{a}$ & II \\
\hline
\end{tabular}

Clinical activity was assessed by attack rate or attack-free status. MRI activity was assessed by Gd-enhancement, new T2 lesions, or both. Clinical severity was assessed by confirmed Expanded Disability Status Scale progression. MRI severity was assessed by total T2 volume (burden) of disease. Merged cell indicates that groups were combined for the purpose of statistical analysis (post hoc method done to increase statistical power).

* Trials of more than 2 years' duration.

$\dagger p<0.05 ; \ddagger p<0.01 ; \S p<0.001$.

a Significant only on interval analysis method ( $p=0.03$ ); not significant on the anytime positive method.

b Analysis using combined data from Avonex-, Betaseron-, and Rebif-treated patients.

c These analyses have been made by combining the findings from the low-dose and high-dose arms of this trial.

$\mathrm{n}=$ number of neutralizing antibody (NAb)-positive patients studied; + = outcome worse in NAb-positive group than NAb-negative group; - = outcome worse in NAb-negative group than NAb-positive group; ? = actual outcome not reported; blank cells = no information provided; NS = not significant; MS = multiple sclerosis; MSCRG = Multiple Sclerosis Collaborative Research Group; INCOMIN = Independent Comparison of Interferon.

sessment (available at www.neurology.org). The questions posed by this assessment are as follows: 1) Once NAb-positivity has developed in an individual patient, does this state persist? 2) Are NAbs to IFN $\beta$ associated with a reduced effectiveness in IFN $\beta$ treated patients with respect to the activity or the severity of MS (measured either clinically or radiographically)? 3) Does the prevalence of NAbpositivity (i.e., the seroprevalence) differ between the different IFN $\beta$ products?

Methods. A panel of neurologists analyzed the evidence relating to NAbs using a literature search with the key words antibodies and interferon beta. We used the MEDLINE database from 1966 to 2005. In addition, the reference lists of the articles identified were reviewed to identify articles not found by the computer search. Using these methods we identified 627 articles. Twentyseven articles in the English language reporting clinical or radiographic outcomes in both antibody positive and antibody negative patients were reviewed. ${ }^{14-41}$ The entire panel classified the level of evidence provided by each article. Several studies were classified as providing Class II evidence (table 1), despite a randomized placebo-controlled trial design (RCTs). This is because evidence associated with NAb status is always post hoc and because patients can never be randomized with respect to their ultimate NAb status. Therefore, one can never exclude the possibility that there are patient-specific factors, which both predispose certain patients to the development of NAbs and, in an unrelated manner, make them either more or less susceptible to MS attacks. If so, this will make NAbs artificially appear to increase or decrease the MS attack rate, underscoring the fact that evidence of an association cannot prove causation.

Detecting and measuring antibodies to IFN $\beta$. Antibodies to IFN $\beta$ ultimately develop in many IFN $\beta$-treated patients. ${ }^{14-41}$ Two classes of antibodies are recognized. Binding antibodies (BAbs) may or may not interfere with IFN $\beta$ function while neutralizing antibodies (NAbs) interfere with IFN $\beta$ function in vitro, presumably by altering (or blocking) binding to the $I F N \alpha / \beta$ receptor. Conceptualized in this manner, NAbs are a subset of the BAbs. Nevertheless, this conception may be simplistic. For example, in a recently presented study, ${ }^{42}$ some NAb-positivity was measured in patients who were BAb-negative. Because only the BAb assay is specific for IgG, and if there is not some technical reason for this result, this suggests that some measured NAb-positivity may not be antibody mediated.

IFN $\beta$ antibodies can be detected through ${ }^{28,43-46}$ binding assays, including enzyme-linked immunosorbent assays (ELISAs) and radio-immuno-precipitation assays (RIPAs), that measure all BAbs. ${ }^{28,46}$ Two assays specifically measure NAbs. The cytopathic effect $(\mathrm{CPE})$ assay measures a reduction in the amount of IFN $\beta$ induced inhibition of virally mediated cell lysis. ${ }^{43}$ By contrast, the MxA assay measures a reduction (either in vitro or in vivo) in the amount of IFN $\beta$-induced MxA protein (or mRNA) synthesis. ${ }^{44,45}$ 
Both the CPE and the MxA assays depend upon assay conditions and require standardization. Either assay had a 2 to $4 \%$ false positive rate as judged by the other in a clinical trial setting. ${ }^{32}$ For cost reasons, antibodies are often measured using a two-step method, in which sera is screened by a binding assay for the presence of BAbs, and, if positive, assayed for NAbs using the CPE or MxA methods. ${ }^{28}$

It is possible that NAbs attach to the receptor-binding region of the IFN $\beta$ molecule, whereas non-NAbs attach to less critical epitopes. Some BAbs seem to have little measurable impact on IFN $\beta$ activity (as it is measured by current NAb assays) although BAbs still might lower serum IFN $\beta$ levels by increasing IFN $\beta$ clearance through the reticuloendothelial system. Despite the fact that in vivo IFN $\beta$ activity might be affected in these alternative ways, NAbs are associated with attenuation of many IFN $\beta$ induced proteins, including IFN $\beta$-specific proteins such as TRAIL (see supplementary data on the Neurology Web site at www.neurology.org). ${ }^{47-49}$

Determining NAb-positivity. Varying definitions of NAbpositivity make comparisons between studies problematic. Many use an arbitrary titer of 20 neutralizing units (NU) per milliliter as the cutoff value for NAb-positivity, although there is evidence that higher titers (e.g., more than 100 or $200 \mathrm{NU} / \mathrm{mL}$ ) are more likely to have an impact on clinical parameters and biomarkers than lower titers. ${ }^{29,31,32,41,48,49}$ Additionally, some patients revert from NAb-positive to NAb-negative status over time. Reversion is more likely with NAb titers of less than $200 \mathrm{NU} / \mathrm{mL}$, although it can happen at titers as high as 3,094 NU/mL. 31,35

In analyzing the effects of NAbs, some authors have used the ever positive, always positive method, in which patients who were ever NAb-positive are compared to persistently NAb-negative subjects..$^{19,21-25}$ Other studies use the so-called once positive, always positive method, in which only observations after the patient has become NAb-positive (often defined as two consecutive positive tiers) are compared to observations in NAb-negative subjects. ${ }^{14-16,20,29,31}$ Each of these methods fails to account for subjects who revert to NAb-negative status after becoming $\mathrm{NAb}$-positive. In a reanalysis of the IFN $\beta-1 b$ (Betaseron) trial, $51 \%$ to $65 \%$ of the NAb-positive patients in the high-dose arm reverted to NAb-negative status at some time. ${ }^{32}$ Inevitably, clinical attacks occurring during a patient's NAb-negative periods will be attributed inappropriately to the attack rate in the NAb-positive group. Attempts to minimize such errors include measuring NAbs every 3 months and assuming that the switch in NAb status occurred at the time of NAb measurement, ${ }^{29,32}$ or measuring NAbs at 12 -month intervals, presuming subjects were at the measured NAb status from 6 months preceding to 6 months following the NAb determination..$^{30}$ Recently, in the so-called interval analysis method, investigators assumed subjects were NAb-positive throughout a 6-month interval if they were positive (by a single determination) at the end of the interval. ${ }^{38}$ Unfortunately, each of these analysis methods will combine data from both NAb-positive and NAb-negative periods when they are used in a population of patients who are spontaneously switching their NAb status. This will be an even greater problem if there is a delay in the clinical impact of NAbs, as suggested by some authors. Most importantly, however, these alternative methods have not particularly clarified the clinical impact of NAbs when compared directly to either the once positive, always positive or the ever positive, always positive methods. ${ }^{29,30,32,35}$

Due to small numbers (table 1), data often lack the statistical power to detect a convincing effect of NAbs. ${ }^{14-18,25,37,38,40,41}$ Sometimes, two different treatment arms of a trial are combined in an attempt to increase statistical power. ${ }^{35,38}$ Because such analyses are done post hoc, they increase the likelihood of bias. Unfortunately, because so little post-marketing information has been acquired, we continue to lack studies with sufficient statistical power to address many important NAb questions, despite more than 150,000 patients being on IFN $\beta$ therapy worldwide.

\section{Analysis of the evidence.}

General considerations regarding the evidence. As an example of the potential impact of NAbs on IFN $\beta$ efficacy, $38 \%$ of patients in the high-dose arm of the phase III IFN $\beta-1 b$ (Betaseron) trial ${ }^{10,14,16}$ be- came NAb-positive (defined as two consecutive positive titers of $\geq 20 \mathrm{NU} / \mathrm{mL} 3$ months apart and also as once positive, always positive) after 2 years. When NAb-positive and NAb-negative patients were analyzed separately, the NAb-positive patients seemed to have attack rates similar to placebo-treated patients. ${ }^{10}$ There are several reasons why such an observation might not be interpreted as easily as it might seem at first glance and also why, in general, relating NAb titers to outcome (either positive or negative) is fraught with problems.

First, as discussed earlier, many of the patients analyzed in this fashion did not become NAb-positive until late in the course of the trial when attack rates had spontaneously declined in all treatment groups. ${ }^{32}$ Even among those patients who became NAb-positive in this study, a large percentage ultimately became NAb-negative, at least temporarily. ${ }^{32}$ Both of these circumstances confound any straightforward interpretation of the data.

Second, the relationship of the IFN $\beta$ activities neutralized by NAbs to the mechanisms whereby IFN $\beta$ exerts its therapeutic effects in MS is uncertain. Although it seems reasonable to expect that interference with one receptor-mediated action would translate to an impact on all such actions, this may not be the case. For example, as noted in the supplementary data (www.neurology.org), despite the fact that IFN $\alpha$ and IFN $\beta$ bind to the same receptor, each molecule has a distinct pattern of downstream biologic effects. ${ }^{2,5}$ Consequently, receptor binding could be distorted by NAbs in such a way that some functions, but not others, are impacted.

Third, antigen-antibody complexes (and thus the mere presence of BAbs with or without NAbs) can also modulate immune functions. These effects will be independent of the receptor-mediated functions of IFN $\beta$ and would be expected to be most conspicuous in patients with high antibody titers.

Fourth, because of the marked intersubject variability in both the clinical course of MS and the response to administered IFN $\beta$, and because IFN $\beta$ has only a modest effect on clinical outcomes, it will be very difficult to establish conclusively a deleterious effect of NAbs on clinical outcomes using small datasets. To do this will require the study of large numbers of NAb-positive patients although, with over 150,000 patients on therapy worldwide, these numbers should be easily achievable.

Once NAb-positivity has developed in an individual patient, does this state persist? Studies of the natural history of NAbs in IFN $\beta$-treated patients suggest that the NAb-positive state is often transient. For example, in a subset of patients from the original IFN $\beta-1 b$ trial, almost $80 \%$ of NAb-positive patients had reverted to NAb-negative status after 8 years ${ }^{12}$ despite continued IFN $\beta-1 b$ therapy. Also, as mentioned earlier, 51 to $65 \%$ of NAb-positive patients in the high dose arm of this trial reverted to $\mathrm{NAb}$-negative status (at least temporarily) within the first 3 years. ${ }^{32}$ Similarly, in a recent study of 23 
NAb-positive IFN $\beta$ - $1 b$ treated patients who were switched to IFN $\beta-1$ a, only 6 out of $20(30 \%)$ and 3 out of $14(21 \%)$ remained NAb-positive after 2 and 5 years. ${ }^{50}$ Also, the reversion rate from NAb-positive to NAb-negative status was $50 \%$ in a small Italian study after 3 to 4 years ${ }^{41}$ and it was $54 \%$ after 3 years in the North American trial of IFN $\beta-1 b$ in SPMS. ${ }^{33}$ It seems that, despite continued treatment with IFN $\beta$, the majority of NAb-positive patients will ultimately revert to NAb-negative status after 3 to 8 years of therapy. Nevertheless, the actual rate of NAb disappearance is difficult to define precisely because the data from long-term studies may be biased from the potential impact of selective drop-out (i.e., patients doing poorly on therapy will stop). This apparently increased tolerance to IFN $\beta$ over time may be molecule specific. In a Danish study of 455 patients, the authors reported that the cumulative probability of reverting to definitely NAb-negative status (i.e., two consecutive NAb-negative titers) in IFN $\beta-1 b$ (Betaseron) treated patients was 57\% after 42 months (CI $=0.43$ to 0.71 ) compared to only $19 \%$ $(\mathrm{CI}=0.07$ to 0.30$)$ in IFN $\beta-1 \mathrm{a}$ (Rebif) treated patients over the same time period. ${ }^{13}$ Because most patients who revert to NAb-negative status tend to have titers of $100 \mathrm{NU} / \mathrm{mL}$ or less ${ }^{29,32,38,41,48,49}$ such a difference might reflect higher NAb-titers to IFN $\beta-1 a$ compared to IFN $\beta-1 b .{ }^{51}$

Are NAbs to IFN $\beta$ associated with an increase in the activity or the severity of MS (measured either clinically or radiographically) in IFN $\beta$-treated patients? Persistently high NAb titers to IFN $\beta$ seem likely to have an impact on the clinical and radiographic efficacy of IFN $\beta$, particularly as assessed by MRI (table 1). The effect of NAbs on clinical measures (especially measures of disease severity such as confirmed Expanded Disability Status Scale progression) is less convincing, although, even for clinical measures of disease activity (i.e., attack rate), the majority of studies greater than 2 years in duration reported a higher attack rate in NAb-positive compared to NAb-negative patients (table 1). Thus, in the larger (or longer) trials, such as the PRISMS, , 21,22,35 the European SPMS, ${ }^{20,29}$ and the North American SPMS studies, ${ }^{33}$ a NAb-associated increase in relapse rate was found ( $p=0.05$ to 0.01 ).

Impact of NAb-positivity on clinical decisions. Despite this evidence, however, it is still unclear whether NAbs eliminate or merely attenuate the effect of IFN $\beta$. Some individuals can have an apparently excellent response to IFN $\beta$ despite having very high NAb titers. For example, in an NIH study of IFN $\beta-1 b, 3$ of the 11 patients with NAbs had both titers $>400 \mathrm{NU} / \mathrm{mL}$ (including the patient with the second highest NAb titer of $1044 \mathrm{NU} / \mathrm{mL}$ ) and a greater than $90 \%$ suppression of MRI activity over the 36 months following the start of IFN $\beta-1 b .{ }^{31}$ In a recent bioactivity study ${ }^{52}$ the authors reported that, despite a marked reduction of the normalized ratio (NR) for in vivo IFN $\beta$-induced MxA production, the $\mathrm{NR}$ was still greater than the normal mean of 1.0 in most ( $82 \%)$ of the NAb-positive patients and, in over half $(65 \%)$, the NR was more than three times normal even with NAb-titers up to $800 \mathrm{NU} / \mathrm{mL} .{ }^{52}$ Thus, although the IFN $\beta$ effect on MxA was attenuated by NAbs, it was not completely eliminated in most patients. ${ }^{52}$ Because it is unknown whether such a low level of MxA induction is associated with continued clinical benefit, it is also unknown whether it would be wise to switch a NAb-positive patient to a noninterferon when they are otherwise clinically well. Indeed, because of our uncertainty about the relationship of $\mathrm{MxA}$ induction to the mechanisms of IFN $\beta$ benefit, because of the variability of the clinical data (table 1), and because there is persistent MxA mRNA expression or MxA induction in some persistently NAb-positive individuals, ${ }^{48,49,52}$ this course of action cannot be recommended. In a NAb-positive patient doing poorly, an alternative therapy should be considered, although such a course of action should probably be considered anyway, regardless of the patient's NAb status. Because $\mathrm{NAb}$-status might influence the choice of subsequent therapy, well standardized and easily accessible methods for NAb measurement should be available to practicing clinicians. Nevertheless, a cautious interpretation by treating neurologists (considering both clinical and probably also MRI data) is necessary.

It is also uncertain whether the apparently deleterious effect of NAbs is offset by the improved efficacy reported with high-dose (more frequently administered) IFN $\beta .^{8}$ There are only two randomized headto-head comparative trials which might conceivably answer such a question. These are the 63-week EVIDENCE trial, ${ }^{26,27}$ which provides Class I comparative data for both clinical and MRI outcomes, and the 2-year Independent Comparison of Interferon (INCOMIN) trial, ${ }^{25}$ which provides Class I comparative data for MRI outcomes and Class III data for clinical outcomes. Both trials, particularly EVIDENCE, are too short to provide a complete answer in view of the dynamics of NAb-positivity discussed earlier. In both trials, NAb-positive patients (defined as positive after a single positive titer of more than $20 \mathrm{NU} / \mathrm{mL}$ ) in the high-dose (more frequent) IFN $\beta$ arms had lower relapse rates and less MRI activity than the arm receiving low-dose (once weekly) IFN $\beta$ regardless of their NAb status. ${ }^{25-27,39}$ Therefore, within the first 2 years of treatment, the available evidence favors using the more effective therapy, even if this therapy is associated with a greater seroprevalence of NAbs. Whether the relative advantage of high-dose (more frequently administered) therapy is sustained beyond 2 years is unknown, but any such consideration of long-term impact must estimate and take into account both the magnitude of the NAb-effect and the probability of (and time course for) the spontaneous disappearance of NAbs, which occurs in many patients. ${ }^{12,13,25,31,32,50}$

Does the rate of $N A b$ production differ between the different IFN $\beta$ products? Prevalence data for NAbs 


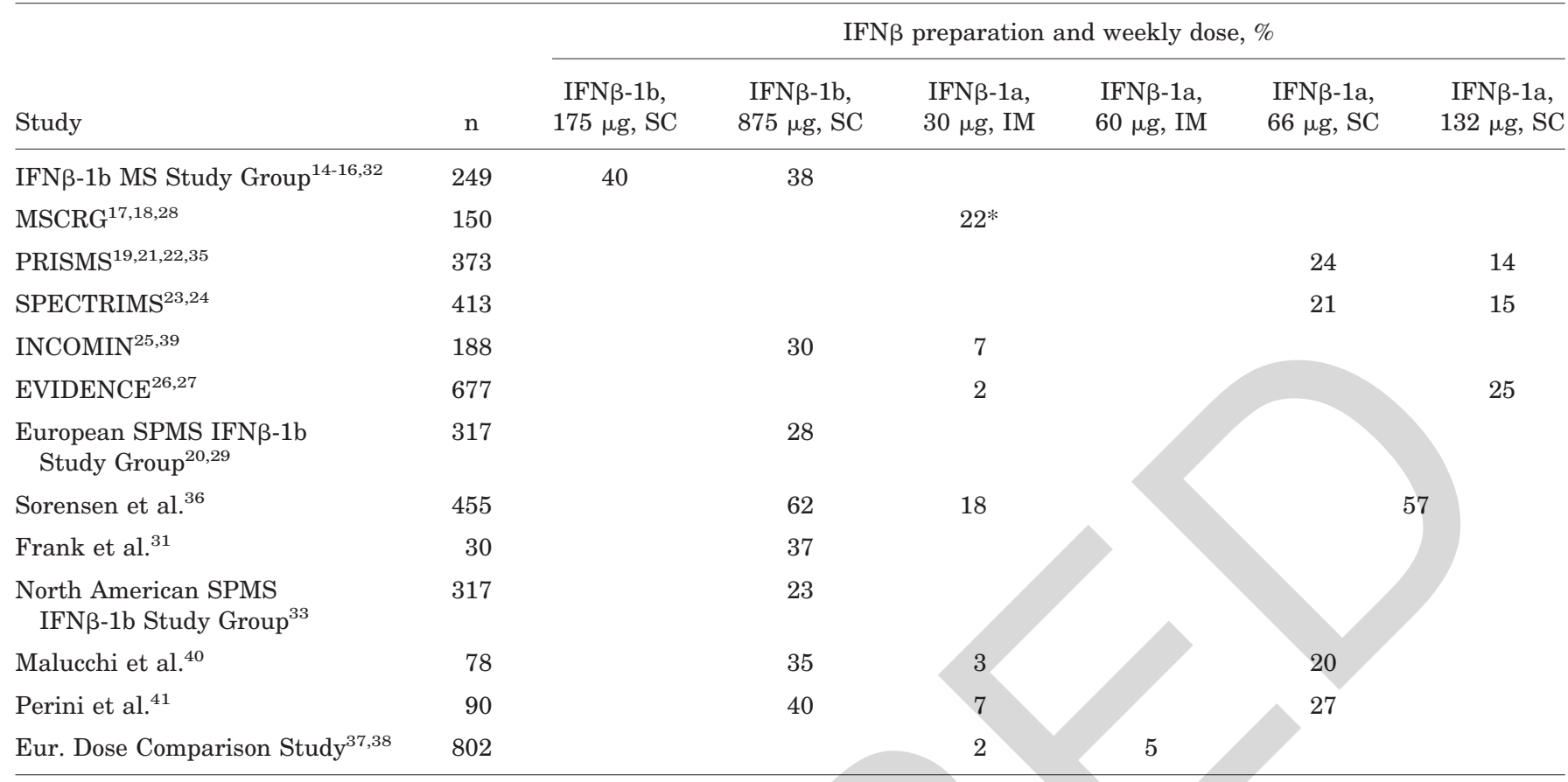

$\mathrm{n}=$ total number of IFN $\beta$-treated patients studied in one or more of the listed dosage arms. Numbers rounded to the nearest $1 \%$.

* Data from a differently formulated product from that which is currently available.

NAbs = neutralizing antibodies; MSCRG = Multiple Sclerosis Collaborative Research Group; INCOMIN = Independent Comparison of Interferon.

(table 2) is confounded by nonuniform definition of the NAb-positive state and by differences in the assays used. In the Multiple Sclerosis Collaborative Research Group (MSCRG) trial of IFN $\beta-1 \mathrm{a}$ (Avonex), 22\% of patients developed NAbs (defined as always positive on the basis of a single titer $\geq 20 \mathrm{NU} / \mathrm{mL}$ ) after 2 years of therapy. ${ }^{17,18,28}$ By contrast, using a newly formulated product, NAbs have generally been found in $7 \%$ or less of the IFN $\beta$-1a treated patients. ${ }^{25,26,28,37-41,48,53}$ The reason for the difference in seroprevalence between formulations is unknown and demonstrates that efficacy for reformulated products requires a clinical study, not merely an inference from studies using previous formulations.

The seroprevalence of NAbs IFN $\beta-1 b$ seems higher than with IFN $\beta-1 \mathrm{a}$ (table 2). ${ }^{10,14,16,25,28}$ However, in a recent survey of 6,698 patients with MS on IFN $\beta-1 b$ therapy, ${ }^{54}$ the seroprevalence of NAbs (defined as a single positive test with a titer $\geq 20 \mathrm{NU} / \mathrm{mL}$ ) in two clinically deteriorating cohorts (21\% in North America and $28 \%$ in Europe) was significantly lower $\left(p<10^{-28}\right.$ for North America and $p<10^{11}$ for Europe) compared to NAb seroprevalence in an unselected cohort (37\% in Australia). Although these unexpected results raise serious questions about any posited connection between $\mathrm{NAb}$-positivity and reduced efficacy, the findings need replication in a more controlled setting before any strong conclusions can be drawn.

With respect to the effect of dose on seroprevalence, two studies demonstrated greater NAb- positivity among low-dose than high-dose arms. ${ }^{19,21-24}$ A follow-up trial of placebo patients re-randomized to low or high dose IFNbeta-1a did not confirm this result (table 2). To further confuse matters, the EVIDENCE trial found NAb-positivity in $25 \%$ of highdose Rebif-treated patients after 1 year, ${ }^{26,27}$ a number almost double that in earlier placebocontrolled trials..$^{19,21-24}$ By contrast, in the European dose-comparison study of IFN $\beta-1 \mathrm{a}$ IM, ${ }^{37,38}$ the $60-\mu \mathrm{g}$ dose resulted in almost three times the seroprevalence of NAbs compared to the $30-\mu \mathrm{g}$ dose. Despite these conflicting observations, it seems that IFN $\beta-1 \mathrm{a}$ is probably less immunogenic than IFN $\beta-1 b$, especially when administered IM. This could be the result of molecular structure. IFN $\beta-1 a$ is glycosylated, which may reduce its immunogenicity compared to the non-glycosylated IFN $\beta-1 b .{ }^{55-57}$ Also, if IFN $\beta-1 b$ forms aggregates, this may increase immunogenicity. Possibly, differences in IFN $\beta$ solubility (caused by the different physical properties of the molecules) or the subcutaneous route might predispose to $\mathrm{NAb}$ formation. Regardless, the randomized EVIDENCE trial, ${ }^{26,27}$ which found a marked difference in $\mathrm{NAb}$ prevalence between Avonex and Rebif (2\% and 25\%), indicates that the dose, the formulation, the route, or the frequency of IFN $\beta-1 \mathrm{a}$ administration make an important difference.

Conclusions. 1. Treatment of MS with IFN $\beta$ (Avonex, Betaseron, or Rebif) is associated with the production of NAbs to the IFN $\beta$ molecule (Level A). 
2. It is probable that the presence of NAbs, especially in persistently high titers, is associated with a reduction in the radiographic and clinical effectiveness of IFN $\beta$ treatment (Level B).

3 . It is probable that the rate of NAb production is less with IFN $\beta$-1a treatment compared to IFN $\beta-1 b$ treatment (Level B). However, because of the variability of the prevalence data, and because NAbs disappear in the majority of patients even with continued treatment (especially in those with low-titer NAbs), the magnitude and persistence of any difference in seroprevalence between these forms of IFN $\beta$ is difficult to determine.

4. It is probable that the seroprevalence of NAbs to IFN $\beta$ is affected by one or more of the following: its formulation, dose, route of administration, or frequency of administration (Level B). Regardless of the explanation, it seems clear that IFN $\beta-1 \mathrm{a}$ (as it is currently formulated for IM injection) is less immunogenic than the current IFN $\beta$ preparations (either IFN $\beta-1 a$ or IFN $\beta-1 b)$ given multiple times per week subcutaneously (Level A). Because NAbs may disappear in many patients with continued therapy, the persistence of this difference is difficult to determine (Level B).

5. Although the finding of sustained high-titer NAbs ( $>100$ to $200 \mathrm{NU} / \mathrm{mL}$ ) has been associated with a reduction in the therapeutic effects of IFN $\beta$ on radiographic and clinical measures of MS disease activity, there is insufficient information on the utilization of NAb testing to provide specific recommendations regarding when to test, which test to use, how many tests are necessary, and which cutoff titer to apply (Level U).

Recommendations for future research. 1. In order to incorporate NAb testing into clinical practice, future research must specifically address issues such as the assay system applied and the stratification of risk for losing IFN $\beta$-efficacy based on the degree of test abnormality. Despite this need for further research, much is already known. NAbs generally develop between 6 and 24 months after the onset of therapy, and if NAbs have not developed by this time, they are unlikely to develop in the future. Newer methods of analysis (e.g., measuring the IFN $\beta$-induced in vivo production of $\mathrm{MxA}$ protein or measuring the amount of IFN $\beta$-induced MxA-mRNA expression) may offer more reliable test results. The utility, sensitivity, and specificity for each of these newer techniques for characterizing the in vivo effects of IFN $\beta$ (either in the presence of NAbs or between individuals at baseline) and correlating these changes (or between-subject differences) in the bioactivity of IFN $\beta$ with its subsequent clinical and radiographic actions must be determined.

2 . The methods of NAb measurement need to be standardized in order to facilitate cross-trial comparisons. Patients with persistent NAb titers of more than $200 \mathrm{NU} / \mathrm{mL}$, those with persistent lower titers, and those who change status during the course of a trial need to have their clinical and MRI statuses analyzed separately, and only from the time of their first NAb-positive test result. These patient-groups should be compared to persistently NAb-negative patients (adjusted to the time at which the comparator group first became NAb-positive). The effects of NAbs in patients using different products or different doses of IFN $\beta$ need to be analyzed separately.

3. Future clinical trials need to include a longterm ascertainment of NAb status and its clinical impact.

4. Future clinical trials need to include a determination of IFN-responsiveness in individuals at study onset in order to link the biologic activity in both $\mathrm{NAb}$-positive and NAb-negative groups with clinical and radiographic outcomes.

5. Because of the small number of NAb-positive patients generally available in RCTs, and because patients cannot be randomized with respect to their ultimate NAb status (e.g., table 1), conclusive data will need to be compiled from large-scale postmarketing surveys. The pharmaceutical industry and the physician community need to work together to acquire and share postmarketing surveillance data so as to characterize accurately the prevalence, persistence, and consequence of NAbs.

Mission statement of TTA. The Therapeutics and Technology Assessment Subcommittee (TTA) oversees the development of AAN technology assessments and therapeutic assessments, which are evidence-based statements that assess the safety, utility, and effectiveness of new, emerging, or established therapeutic agents or technologies in the field of neurology. Technology assessments and therapeutic assessments are developed through a rigorous process of defining the topic, evaluating and rating the quality of the evidence, and translating the conclusions of the evidence into practical assessments that can be used to guide the use of technologies and therapeutic agents in the practice of neurology.

Disclaimer. This statement is provided as an educational service of the American Academy of Neurology. It is based on an assessment of current scientific and clinical information. It is not intended to include all possible proper methods of care for a particular neurologic problem or all legitimate criteria for choosing to use a specific procedure. Neither is it intended to exclude any reasonable alternative methodologies. The AAN recognizes that specific patient care decisions are the prerogative of the patient and the physician caring for the patient, based on all of the circumstances involved.

Conflict of interest statement. The American Academy of Neurology is committed to producing independent, critical and truthful clinical practice guidelines (CPGs). Significant efforts are made to minimize the potential for conflicts of interest to influence the recommendations of this CPG. To the extent possible, the AAN keeps separate those who 
have a financial stake in the success or failure of the products appraised in the CPGs and the developers of the guidelines. Conflict of interest forms were obtained from all authors and reviewed by an oversight committee prior to project initiation. AAN limits the participation of authors with substantial conflicts of interest. The AAN forbids commercial participation in, or funding of, guideline projects. Drafts of the guideline have been reviewed by at least three AAN committees, a network of neurologists, Neurology peer reviewers, and representatives from related fields. The AAN Guideline Author Conflict of Interest Policy can be viewed at www.aan.com. With regards to this specific report, all authors have stated that they have nothing to disclose.

\section{Appendix 1}

Therapeutics and Technology Assessment subcommittee members: Janis Miyasaki, MD, FAAN (Co-chair); Yuen T. So, MD, PhD (Co-Chair); Carme Armon, MD, MHS, FAAN (ex-officio); Vinay Chaudhry, MD, FAAN; Richard M. Dubinsky, MD, MPH, FAAN; Douglas S. Goodin, MD (ex-officio, facilitator); Mark Hallett, MD, FAAN; Cynthia L. Harden, MD; Kenneth J. Mack, MD, PhD; Fenwick T. Nichols III, MD; Michael A. Sloan, MD, MS, FAAN; James C. Stevens, MD; Paul W. O’Connor, MD, FAAN.

\section{Appendix 2}

AAN classification of evidence for therapeutic intervention

Class I. Prospective, randomized, controlled clinical trial with masked outcome assessment, in a representative population. The following are required: a) primary outcome(s) clearly defined; b) exclusion/inclusion criteria clearly defined; c) adequate accounting for dropouts and cross-overs with numbers sufficiently low to have minimal potential for bias; and d) relevant baseline characteristics are presented and substantially equivalent among treatment groups or there is appropriate statistical adjustment for differences.

Class II. Prospective matched group cohort study in a representative population with masked outcome assessment that meets a-d above OR a RCT in a representative population that lacks one criteria $a-d$.

Class III. All other controlled trials (including well-defined natural history controls or patients serving as own controls) in a representative population, where outcome is independently assessed, or independently derived by objective outcome measurement*

Class IV. Evidence from uncontrolled studies, case series, case reports, or expert opinion.

\footnotetext{
* Objective outcome measurement: an outcome measure that is unlikely to be affected by an observer's (patient, treating physician, investigator) expectation or bias (e.g. blood tests, administrative outcome data).
}

\section{Appendix 3}

\section{Classification of recommendations}

$\mathrm{A}=$ Established as effective, ineffective, or harmful for the given condition in the specified population. (Level A rating requires at least two consistent Class I studies.)

$\mathrm{B}=$ Probably effective, ineffective, or harmful for the given condition in the specified population. (Level B rating requires at least one Class I study or at least two consistent Class II studies.)

$\mathrm{C}=$ Possibly effective, ineffective, or harmful for the given condition in the specified population. (Level $\mathrm{C}$ rating requires at least one Class II study or two consistent Class III studies.)

$\mathrm{U}=$ data inadequate or conflicting; given current knowledge, treatment is unproven.

\section{References}

1. Came PE, Carter WA, eds. Interferons and their applications. Berlin: Springer-Verlag; 1984.

2. Der SD, Zhou A, Williams BRG, Silverman RH. Identification of genes differentially regulated by interferon $\alpha, \beta$, or $\gamma$ using oligonucleotide arrays. Proc Natl Acad Sci USA 1998;95:15623-15628.

3. Reder AT, ed. Interferon therapy of multiple sclerosis. New York: Marcel Dekker, Inc.; 2000.
4. Goodbourn S, Didcock L, Randall RE. Interferons: cell signaling, im mune modulation, antiviral responses and virus countermeasures. J Gen Virol 2000;81:2341-2364.

5. de Veer MJ, Holko M, Frevel M, et al. Functional classification of interferon-stimulated genes identified using microarrays. J Leukoc Biol 2001;69:912-920.

6. Servant MJ, Tenoever B, Lin R. Overlapping and distinct mechanisms regulating IRF-3 and IRF-7 function. J Interferon Cytokine Res 2002 22:49-58

7. Kerr IM, Costa-Pereira AP, Lillemeier BF, Strobl B. Of JAKs, STATs, blind watchmakers, jeeps and trains. FEBS Lett 2003;546:1-5.

8. Goodin DS, Frohman EM, Garmany GP Jr, et al. Disease modifying therapies in multiple sclerosis. Report of the Therapeutics and Technology Assessment Subcommittee of the American Academy of Neurology and the MS Council for Clinical Practice Guidelines. Neurology 2002; 58:169-178.

9. Stürzebecher S, Maibauer R, Heuner A, Beckmann K, Aufdembrincke B. Pharmacodynamic comparison of single doses of IFN $\beta-1 \mathrm{a}$ and IFN $\beta-1 b$ in healthy volunteers. J Interferon Cytokine Res 1999;19: 1257-1264

10. IFNB Multiple Sclerosis Study Group and the UBC MS/MRI Analysis Group. Neutralizing antibodies during treatment of multiple sclerosis with interferon beta-1b: experience during the first three years. Neurology 1996;47:889-894.

11. Ross C, Clemmesen KM, Svenson M, et al., and the Danish Multiple Sclerosis Study Group. Interferon-beta in multiple sclerosis patients: influence of preparation, dosage, dose frequency, and route of administration. Ann Neurol 2000;8:706-712.

12. Rice GP, Paszner B, Oger J, et al. The evolution of neutralizing antibod ies in multiple sclerosis patients treated with interferon $\beta-1 b$. Neurology 1999;52:1277-1279.

13. Sorensen PS, Koch-Henriksen N, Ross C, et al. , and the Danish Multiple Sclerosis Study Group. Appearance and disappearance of neutralizing antibodies during interferon-beta therapy. Neurology 2005;65:3339

14. The IFNB Multiple Sclerosis Study Group. Interferon beta-1b is effective in relapsing-remitting multiple sclerosis. I. Clinical results of a multicenter, randomized, double-blind, placebo-controlled trial. Neurology 1993;43:655-661.

15. Paty DW, Li DKB. The UBC MS/MRI Study Group, and the IFNB Multiple Sclerosis Study Group. Interferon beta-1b is effective in relapsing-remitting multiple sclerosis. II. MRI analysis results of a multicenter, randomized, double-blind, placebo-controlled trial. Neurology 1993;43:655-661.

16. The IFNB Multiple Sclerosis Study Group and the UBC MS/MRI Analysis Group. Interferon beta-1b in the treatment of multiple sclerosis: final outcome of the randomized controlled trial. Neurology 1995;45: 1277-1285.

17. Jacobs LD, Cookfair DL, Rudick RA, et al. , and the Multiple Sclerosis Collaborative Research Group (MSCRG). Intramuscular interferon beta-1a for disease progression in relapsing multiple sclerosis. Ann Neurol 1996;39:285-294.

18. Simon JH, Jacobs LD, Campion M, et al., and the Multiple Sclerosis Collaborative Research Group (MSCRG). Magnetic resonance studies of intramuscular interferon $\beta-1$ a for relapsing multiple sclerosis. Ann Neurol 1996;43:79-87.

19. PRISMS Study Group. Randomized double-blind placebo-controlled study of interferon $\beta$-1a in relapsing-remitting multiple sclerosis. Lancet 1998;352:1498-1504.

20. European Study Group on Interferon $\beta-1 b$ in Secondary Progressive MS. Placebo-controlled multicentre randomised trial of interferon $\beta-1 b$ in treatment of secondary progressive multiple sclerosis. Lancet 1998 352:1491-1497.

21. Li DH, Paty DW. The UBC MS/MRI Analysis Research Group, and the PRISMS Study Group. Magnetic resonance imaging results of the PRISMS trial: a randomized, double-blind, placebo-controlled study of interferon- $\beta 1 \mathrm{a}$ in relapsing-remitting multiple sclerosis. Ann Neurol 1999;46:197-206.

22. PRISMS Study Group and the UBC MS/MRI Analysis Group. PRISMS-4: Long-term efficacy of interferon-beta-1a in relapsing MS. Neurology 2001;56:1628-1636.

23. SPECTRIMS Study Group. Randomized controlled trial of interferonbeta-1a in secondary progressive MS: clinical results. Neurology 2001; $56: 1496-1504$.

24. Li DK, Zhao GJ, Paty DW. The UBC MS/MRI Analysis Research Group, and the SPECTRIMS Study Group. Randomized controlled trial of interferon-beta-1a in secondary progressive MS: MRI results. Neurology 2001;56:1505-1513.

25. Durelli L, Verdun E, Barbero P, et al., and the Independent Comparison of Interferon (INCOMIN) Trial Study Group. Every-other-day interferon beta-1b versus once-weekly interferon beta-1a for multiple sclerosis: results of a 2 -year prospective randomised multicentre study (INCOMIN). Lancet 2002;359:1453-1460.

26. Panitch H, Goodin DS, Francis G, et al., the EVIDENCE Study Group, and the UBC MS/MRI Research Group. A randomized, comparative study of interferon beta dose in MS: the EVIDENCE trial. Neurology 2002;59:1496-1506. 
27. Panitch H, Goodin DS, Francis G, et al., the EVIDENCE Study Group and the UBC MS/MRI Research Group. Benefits of high-dose, highfrequency interferon beta-1a in relapsing MS are sustained to 16 months: final comparative results of the EVIDENCE trial. Arch Neurol 2007 (in press)

28. Rudick RA, Simonian NA, Alam JA, et al. Incidence and significance of neutralizing antibodies to interferon beta-1a in multiple sclerosis. Multiple Sclerosis Collaborative Research Group (MSCRG). Neurology 1998;50:1266-1272.

29. Polman C, Kappos L, White R, et al., and the European Study Group in Interferon Beta-1b in Secondary Progressive MS. Neutralizing antibodies during treatment of secondary progressive MS with interferon beta1b. Neurology 2003;60:37-43.

30. Sorensen PS, Ross C, Clemmesen KM, et al., and the Danish Multiple Sclerosis Study Group. Clinical importance of neutralising antibodies against interferon beta in patients with relapsing-remitting multiple sclerosis. Lancet 2003;362:1184-1191.

31. Frank JA, Richert N, Bash C, et al. Interferon-beta-1b slows progression of atrophy in RRMS: three-year follow-up in NAb- and NAb+ patients. Neurology 2004;62:719-725.

32. Petkau AJ, White RA, Ebers GC, et al., and the IFNB Multiple Sclerosis Study Group. Longitudinal analyses of the effects of neutralizing antibodies on interferon beta-1b in relapsing-remitting multiple sclerosis. Mult Scler 2004;10:126-138.

33. Panitch H, Miller A, Paty D, et al., and the North American Study Group on Interferon beta-1a in Secondary Progressive MS. Interferon beta-1b in secondary progressive MS: results from a 3-year controlled study. Neurology 2004;63:1788-1795.

34. The IFNB Multiple Sclerosis Study Group. Neutralizing antibodies during treatment of multiple sclerosis with interferon beta-1b. Neurology 1996;47:889-894.

35. Francis GS, Rice GPA, Alsop JC, and the PRISMS Study Group. Interferon $\beta-1 \mathrm{a}$ in MS: Results following development of neutralizing antibodies in PRISMS. Neurology 2005;65:48-55.

36. Soelberg Sorensen P, Koch-Henriksen N, Ross C, et al. , and the Danish Multiple Sclerosis Study Group. Appearance and disappearance of neutralizing antibodies during interferon-beta therapy. Neurology 2005;65: 33-39.

37. Clanet M, Radue EW, Kappos L, the European IFN beta-1a (Avonex) Dose-Comparison Study Investigators, et al. A randomized, doubleblind, dose-comparison study of weekly interferon beta-1a in relapsing MS. Neurology 2002;59:1507-1517.

38. Kappos L, Clanet M, Sandberg-Wollheim, the European Interferon Beta-1a IM Dose-Comparison Study Investigators, et al. Neutralizing antibodies and efficacy in interferon $\beta$-1a: a 4-year controlled study. Neurology 2005;65:40-47.

39. Barbero P, Bergui M, Versino E, et al., and the INCOMIN Trial Study Group. Every-other-day interferon beta-1b versus once-weekly interferon beta-1a for multiple sclerosis (INCOMIN Trial) II: analysis of MRI responses to treatment and correlation with NAb. Mult Scler 2006;12:72-76.

40. Malucchi S, Sala A, Gilli F, et al. Neutralizing antibodies reduce the efficacy of $\beta$ IFN during treatment of multiple sclerosis. Neurology 2004 62:2031-2037.
41. Perini $\mathrm{P}$, Calabrese $\mathrm{M}$. The clinical impact of interferon beta antibodies in relapsing-remitting MS. J Neurol 2004;251:305-309.

42. Gilli F, Sala F, Hoffmann F, et al. Anti-interferon beta neutralizing activity is not entirely mediated by antibodies. Mult Scler 2005 11(suppl 1):S2-3.

43. Grossberg SE, Kawade Y, Kohase M, Klein JP. The neutralization of interferons by antibody. II. Neutralizing antibody unitage and its relationship to bioassay sensitivity: the tenfold reductions unit. J Interferon Cytokine Res 2001;21:743-755.

44. Pachner AR. Anticytokine antibodies in beta interferon-treated MS patients and the need for testing: Plight of the practicing neurologist. Neurology 1997;49:647-650.

45. Pachner AR, ed. Anti-IFN $\beta$ antibodies in IFN $\beta$-treated patients. Neurology 2003;61 (suppl 5):S1-S39.

46. Lawrence N, Oger J, Aziz T, Palace J, Vincent A. A sensitive radioimmunoprecipitation assay for assessing the clinical relevance of antibodies to IFN beta. J Neurol Neurosurg Psychiatry 2003;74:1236-1239.

47. Khan QA, Xia Q, Bever CT, et al. Interferon beta-1b serum levels in multiple sclerosis patients following subcutaneous administration. Neurology 1996;46:1639-1643.

48. Bertolotto A, Gilli F, Sala A, et al. Persistent neutralizing antibodies abolish the interferon $\beta$ bioavailability in MS patients. Neurology 2003; 60:634-639.

49. Bertolotto A. Neutralizing antibodies to interferon beta: implications for the management of multiple sclerosis Curr Opinion Neurol 2004;17: 241-246.

50. Herndon RM, Rudick RA, Munschauer FE, et al. Eight-year and safety of interferon beta-1a-Avonex treatment in patients with multiple sclerosis. Mult Scler 2005;11:409-419.

51. Deisenhammer F, Gneiss C, Reichartseder F, et al. Comparative study of neutralizing antibody titers against the three different interferonbeta preparations using a unique and validated assay. Neurology 2005; 64:A326.

52. Pachner AR, Dail D, Pak E, Narayan K. The importance of measuring IFN $\beta$ bioactivity: monitoring in MS patients and the effect of anti-IFN $\beta$ antibodies. J Neuroimmunol 2005;166:180-188.

53. Jacobs LD, Beck RW, Simon JH, et al. Intramuscular interferon beta-1a therapy initiated during a first demyelinating event in multiple sclerosis. CHAMPS Study Group. N Engl J Med 2000;343:898-904.

54. Goodin DS, Hurwitz B, Noronha A. Neutralizing antibodies to interferon $\beta$-1b are not associated with disease worsening in multiple sclerosis. J Int Med Res 2007;35:173-187.

55. Runkel L, Meier W, Pepinsky RB, et al. Structural and functional differences between glycosylated and non-glycosylated forms of human interferon-beta (IFN-beta). Pharm Res 1998;15:641-649.

56. Hochuli E. Interferon: technical evaluation of interferon-alpha 2a. J Interferon Cytokine Res 1997;17:S15-S21.

57. Wang C, Eufemi M, Turano C, Giartosio A. Influences of the carbohydrate moiety on the stability of glycoproteins. Biochem 1996;35:7299 7307.

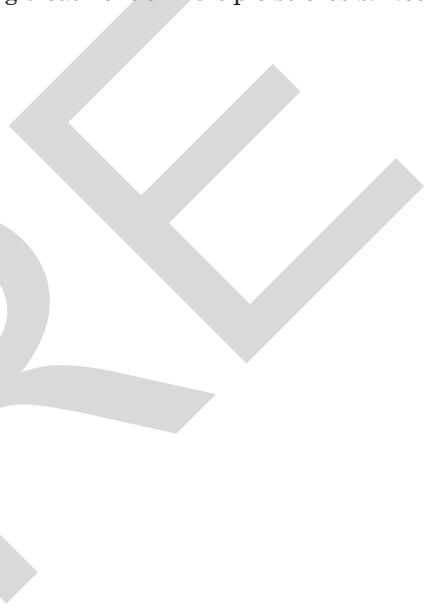

